\title{
Mitigating Tropospheric Propagation Delay Errors in Precise Airborne GPS Navigation
}

\author{
J.P. Collins and R.B. Langley \\ Geodetic Research Laboratory, Department of Geodesy and Geomatics Engineering \\ University of New Brunswick, P.O. Box 4400, Fredericton, N.B. E3B 5A3 Canada
}

\begin{abstract}
The high spatial and temporal variability of the troposphere is well known, as is its effect - through propagation delays - on GPS positioning. This effect can be particularly problematical in airborne kinematic differential positioning where the altitude difference between reference station and aircraft is typically quite large. The use of zenith delay models and mapping functions at ground stations is fairly well understood, however their use for processing data collected on board aircraft is less well understood.
\end{abstract}

Previous tests have indicated that some of the models often used for navigation purposes (e.g. Altshuler, NATO and the proposed WAAS model) perform poorly compared to those generally used for static positioning. These tests were not done under kinematic conditions however, but as comparisons with ray tracing through radiosonde data.

This paper outlines the work recently done at UNB on testing the reliability of tropospheric models in precise airborne GPS navigation. Particular attention has been paid to the performance of the currently proposed WAAS model. The data used to test the models is from an adverse weather flight dynamics experiment undertaken off Newfoundland, Canada, in March 1995. The paper includes an analysis of the GPS flight data to determine the influence of different tropospheric models on the reliability and accuracy of the solutions.

\section{INTRODUCTION}

With the increasing use of the Global Positioning System (GPS) as a primary navigation aid for aircraft of all types, it is important to understand all the potential error sources of the system. These sources are generally well understood after many years of geodetic GPS positioning, however special considerations may exist for airborne positioning.

One of the most significant problems is the effect of the neutral-atmosphere on the electromagnetic signal propagated by a GPS satellite. The effect is to both retard the velocity of the signal and to refract (bend) its raypath. The resulting delay is generally referred to as the tropospheric delay because most of the signal delay occurs in the lowest $10 \mathrm{~km}$ or so of the atmosphere. The effects on geodetic-type static positioning are generally well known, however this is the special case of a signal arriving at an antenna located on the earth's surface. The more general effect on a GPS receiver at arbitrary height above the earth's surface must be considered for airborne positioning.

The particular problems in airborne GPS positioning are: 1) the change of the tropospheric delay with a change in height, and 2) the usual lack of direct meteorological measurements to help quantify the state of the atmosphere. The mitigation of the tropospheric effects will increase the prospect of more accurate and precise airborne GPS positioning. The effects can be two-fold, that is a direct impact on the position calculated from the GPS range measurements and then indirect effects, for example through the incorrect solving of carrier phase ambiguities which then degrades position accuracy. Another possible indirect effect is the contamination of corrections calculated for differential GPS (DGPS) positioning by a reference station.

A primary DGPS-type service that is currently planned for the continental United States and Canada is the Federal Aviation Administration's (FAA) Wide Area Augmentation System (WAAS). Consisting, in its initial phase, of 24 ground reference stations, 2 master stations, 6 ground earth stations and 3 geostationary Inmarsat satellites to broadcast both DGPS corrections and additional GPS-like signals, it represents a considerable investment in the large-scale use of GPS for aircraft positioning [11].

The primary aim of this paper is to examine the potential impact on an aircraft's position caused by using different tropospheric delay models. The models tested are the Altshuler [1], NATO [15] and the currently proposed

\footnotetext{
${ }^{\ddagger}$ Presented at the IEEE Position, Location and Navigation Symposium, Atlanta, Georgia, USA, 22nd - 26th April, 1996
} 
WAAS model (which is derived from Altshuler's [4]). This ongoing research is made possible through the generous support of Transport Canada Aviation.

\section{TROPOSPHERIC DELAY}

An electromagnetic signal propagating through the atmosphere will be affected because the refractive index of the constituent gases is slightly greater than unity. The decrease in velocity increases the time taken for the signal to reach a receiver's antenna, thereby increasing the equivalent path length (both often referred to as the "delay"). At low elevation angles refraction bends the raypath, further increasing the delay.

Assuming that the lower atmosphere is azimuthally symmetric, the effect can be considered in two parts: the delay experienced in the zenith direction and the magnification of the zenith delay that is experienced at other elevation angles. This leads to the common formulation of zenith delays and mapping functions seen in the geodetic GPS literature. [9]:

The tropospheric delay can be defined at the zenith as

$$
d_{\text {trop }}^{z}=\int_{r_{s}}^{r_{a}}[n(r)-1] d r=10^{-6} \int_{r_{s}}^{r_{a}} N d r,
$$

where $\mathrm{n}$ is the refractive index, $\mathrm{N}$ is the refractivity, $\mathrm{r}_{\mathrm{s}}$ is the height of the user's antenna and $r_{a}$ is the height of the "top" of the neutral-atmosphere. The refractivity can be written in two parts, that due to the dry atmospheric gases and that due to water vapour pressure [22]:

$$
N=k_{1}\left(\frac{P_{d}}{T}\right) Z_{d}^{-1}+\left(k_{2} \frac{e}{T}+k_{3} \frac{e}{T^{2}}\right) Z_{w}^{-1},
$$

where $P_{d}$ is the partial pressure of dry air (mbar), $T$ is the absolute temperature $(\mathrm{K}), e$ is the partial pressure of water vapour (mbar) and $Z_{d}$ and $Z_{w}$ are compressibility factors to account for non-ideal gas behaviour [18]. Constants $\mathrm{k}_{1}, \mathrm{k}_{2}$ and $\mathrm{k}_{3}$ are empirically derived. The first term in equation (2) is the so-called "dry" component of refractivity. Using the equation of state, equation (2) can be recast as a function of the total atmospheric density, $\rho$, namely:

$$
N=k_{1} \frac{R}{M_{d}} \rho+\left(k_{2}^{\prime} \frac{e}{T}+k_{3} \frac{e}{T^{2}}\right) Z_{w}^{-1},
$$

where, $k_{2}^{\prime}=\left(k_{2}-k_{1}\left[M_{w} / M_{d}\right]\right), M_{w}$ and $M_{d}$ are the molar masses of water and dry air respectively and $R$ is the molar gas constant. The first term in equation (3) represents the hydrostatic component of refractivity. Combining equations (1) and (3) and ignoring the compressibility factors (which differ from unity by a few parts per thousand at most), we get [7]:

$$
\begin{gathered}
d_{\text {trop }}^{z}=d_{\text {hyd }}^{z}+d_{\text {wet }}^{z} \\
=10^{-6}\left[\left(\frac{k_{1} R}{g_{m} M_{d}}\right) P+\int_{r_{s}}^{r_{a}}\left(k_{2}^{\prime} \frac{e}{T}+k_{3} \frac{e}{T^{2}}\right) d r\right],
\end{gathered}
$$

whereby the assumption of hydrostatic equilibrium in the atmosphere has allowed the integration of the hydrostatic component. Gravity acceleration at the atmospheric column centroid is given by $g_{m}$, a function of latitude and height of the antenna. The hydrostatic zenith delay accounts for about $90 \%$ of the total zenith delay and can be modelled with submillimetre accuracy provided accurate pressure measurements $(P)$ are available [5].

What remains in equation (4) is usually termed the zenith wet delay and can also be integrated after specifying suitable relationships for temperature and water vapour pressure with height. The problem lies in the fact that water vapour is rarely in hydrostatic equilibrium and varies significantly throughout the troposphere. Hence the accuracy of the zenith wet delay as computed by models using surface meteorological measurements is typically no better than several centimetres $[8,13]$.

It then remains to specify functions to map these zenith delays to the elevation angle of the raypath of the arriving signal. Following [5], the typical formulation is:

$$
d_{\text {trop }}=d_{\text {hyd }}^{z} \cdot m_{\text {hyd }}(\varepsilon)+d_{\text {wet }}^{z} \cdot m_{\text {wet }}(\varepsilon),
$$

where the total delay $d_{\text {trop }}$ is a function of the hydrostatic zenith delay $d_{\text {hyd }}^{z}$ and its mapping function $m_{h y d}(\varepsilon)$ and the wet zenith delay $d_{\text {wet }}^{z}$ and its mapping function $m_{\text {wet }}(\varepsilon)$. The elevation angle to the satellite $(\varepsilon)$ is the minimum parameter required, although it is possible to parameterise the mapping functions (as well as the zenith delays) with meteorological and location parameters. Mapping functions derived from ray-tracing inherently take into account the effect of ray bending.

\section{Tropospheric Delay Models}

Some of the first tropospheric delay models developed for space-based positioning techniques did not explicitly separate the zenith delays and mapping functions. The Altshuler model is an example of this type. Several of these models were also constructed to use position and time information only, with no input of meteorological parameters, because of their intended use in airborne 
positioning. Hence both the Altshuler model and the proposed WAAS model derive the tropospheric delay using the station height above sea level, latitude, and the day of year information as inputs. The NATO model requires the input of the user's height.

The NATO model uses an average reference value for surface refractivity which is input into a three-stage equation to give the total tropospheric zenith delay in three layers: 0 to $1 \mathrm{~km}$, between 1 and $9 \mathrm{~km}$ and from $9 \mathrm{~km}$ and above. Although not stated in the reference for this model [15], this zenith delay model is based on the Central Radio Propagation Laboratory (CRPL) Reference Atmosphere1958 (RA-1958) [2]. This model represents atmospheric refractivity as decreasing linearly in the first kilometre above the earth's surface, then exponentially to a constant value of $105 \mathrm{~N}$ units at 9 kilometres and exponentially from there on up.

In the NATO formulation, the total zenith delay is mapped using the Chao [3] dry delay mapping function. The elevation angle dependence in the Altshuler model is modelled using a seven coefficient polynomial, while the proposed WAAS model appears to use only the cosecant of the elevation angle as its mapping function.

The Altshuler model was originally derived from ray tracing through CRPL profiles, including RA-1958, using seasonal world-wide surface refractivity data [1]. With the proposed WAAS model being derived from the Altshuler model, all three of the models we have chosen to test have a similar genesis. It would appear that all three models are also specified with the same mean global surface refractivity value of $324.8 \mathrm{~N}$ units.

For a standard to which these models can be compared, we have chosen the zenith delay models of Saastamoinen [19], which are generally considered the best available (e.g. see [8] and [13]). The corresponding mapping functions we have chosen are those derived by Niell [17] which have been shown to be accurate at very low elevation angles (see [12] for more details). The functions require position and time information only, however they have been shown to perform equally well, and often better than, some mapping functions fully parameterised to work with meteorological values.

The Saastamoinen/Niell model can be driven either with actual measured values of the meteorological parameters or pressure, temperature and water vapour pressure values that are equivalent to the surface refractivity of $324.8 \mathrm{~N}$ units used by those models. Using the refractivity constants quoted in Saastamoinen (those of Essen and Froome [6]) and setting pressure and temperature to the Standard Atmosphere [16] values of 1013.25 mbar and $288.15 \mathrm{~K}$, we derive a water vapour pressure of 11.691 mbar. These values are then mapped to the antenna height by the relationships used to formulate the Saastamoinen delays, namely:

$$
\begin{aligned}
& T=T_{0}-\beta \cdot H, \\
& P=P_{0}\left(\frac{T}{T_{0}}\right)^{\frac{g}{R \beta}}, \\
& e=e_{0}\left(\frac{T}{T_{0}}\right)^{\frac{4 g}{R \beta}},
\end{aligned}
$$

where the subscript " 0 " denotes the mean sea level values quoted above, $\beta$ is the standard atmosphere lapse rate of $0.0065 \mathrm{~K} / \mathrm{m}$ and $\mathrm{H}$ is the antenna height above mean sea level. For the purposes of this paper, we will designate this as the UNB1 model. The first two of these relationships (eq. 6a and 6b) are also specified in the Standard Atmosphere. Strictly speaking these equations are only valid in the troposphere itself. From the tropopause upwards equation (6b) must be used alternately with another pressure equation from the Standard Atmosphere, along with various lapse rate values, to model pressure to the top of the neutral atmosphere. The third relationship is also commonly used in meteorology (see e.g. [21] and [7]).

\section{Flight DAta Description AND PROCESSING}

We are continuing to use the flight data described previously [14] which was collected by the National Research Council of Canada, at and around St. John's, Newfoundland, in March 1995. The GPS data we have processed consists of 12 days of dual-frequency measurements recorded by Ashtech Z-12 receivers located onboard a highly-instrumented Convair-580 aircraft and at a ground reference station in St. John's. The data from each day generally consists of that obtained during one three-to-five hour flight and recorded at a two second sampling interval. Meteorological data was recorded at both the ground station and the aircraft. The ground meteorological data is available at one minute intervals and the airborne data every second.

The flight paths consisted of repeated horizontal and vertical profiles through cloud layers up to heights of approximately $8 \mathrm{~km}$ to investigate the effects of freezing precipitation on aircraft dynamics. Frontal zones and temperature inversions are often associated with potential causes of freezing precipitation and along with the geographic location and unpredictable weather conditions it is expected that the data will provide a good test of the tropospheric delay models. 
The data has been analyzed using the Kinematic and Rapid Static (KARS) software developed by Dr. Gerald Mader at the National Geodetic Survey, National Oceanic and Atmospheric Administration and subsequently modified at UNB. The nature of the flights has forced us to change our processing methods from that used for our previous paper [14]. All of the flights included data when the aircraft was over 100 kilometres from the reference station and on several days the maximum distance was over 600 kilometres. This makes it impractical to use the carrier phase data for our tests. It becomes increasingly difficult to correctly resolve cycle slips or integer ambiguities for new (low elevation) satellites at distances in excess of several tens of kilometres, especially when using the less accurate tropospheric delay models.

The previous paper showed that using a different tropospheric delay model for carrier phase positioning could influence the ambiguity resolution process. In some cases the ambiguities were not only incorrectly resolved, but also at different epochs. Hence, any differencing of solutions to examine the impact of using a different tropospheric delay model could be complicated by spurious jumps and biases due to incorrect integer ambiguities. Therefore, we have chosen to use the $\mathrm{P}(\mathrm{Y})$-code pseudorange measurements that are available. These should still provide precise (compared to C/A code), lownoise measurements. We are once again processing the data using precise International GPS Service for Geodynamics orbits to reduce satellite position errors.

An elevation cut-off angle of five degrees is used to examine the effect of low-elevation measurements. It is important to include these measurements because of the techniques used for verifying system integrity in GPS airborne navigation. These techniques (often designated as RAIM - Receiver Autonomous Integrity Monitoring) generally require more than five satellites in view at all time, unless ground integrity broadcasts from reference stations are also used [10].

It was also intended to use the ionosphere-free linear combination (P3) to remove the ionospheric influence. However, there are occasional data gaps in the $\mathrm{P}(\mathrm{Y})$-code measurements. This problem occurs at both receivers with satellites at low elevation angles, hence it seems that the Ashtech receivers had problems tracking the $\mathrm{P}(\mathrm{Y})$-code signals at these angles. When using the ionosphere-free combination, satellites are temporarily removed from the solution causing spikes in the Position Dilution of Precision (PDOP) indicator and consequent jumps in the solutions. These jumps are exaggerated in the solution differences because they are at low elevation angles where the ray-bending effects may be poorly modelled. While these jumps do not affect our results statistically, they can be confusing. Using the precise L1 pseudorange measurements (P1) and substituting C/A code measurements when required generally gives more consistent PDOP values and is inherently less noisy. Any residual ionospheric influence not removed by the single differencing undertaken during the processing is almost certainly removed when different position solutions are differenced.

To provide a reference with which to compare the impact of the different tropospheric delay models we first of all compute "benchmark" solutions for each day. The benchmark solution uses the Saastamoinen/Niell combination to model the tropospheric delay with inputs from the meteorological data recorded at the aircraft and the ground station. Without an independent way of checking the accuracy of the positions of the aircraft we believe this represents the "best" solution available. Each solution is then sequentially re-computed with each tropospheric delay model substituted for the aircraft data model only. To examine the impact of the model, we difference the solution with the benchmark solution and appraise any differences.

Several previous authors have pointed out the strong correlation of height bias in GPS position solutions and tropospheric delay error (e.g. [23] and [20]). An intuitive understanding of this can be gleaned from considering the hydrostatic delay. Equation (4) shows its fundamental dependence on the atmospheric pressure at the GPS antenna which is conversely dependent, by equations (6a) and (6b), on height. Therefore any attempt to treat the atmosphere at altitude as if it were at the earth's surface, or to ignore the tropospheric effect altogether, will introduce a bias inversely proportional to the height. Accordingly, if the tropospheric models tested here correctly model the structure of the atmosphere with height, then their use of an assumed pressure should only introduce a constant bias when compared to the benchmark solution.

\section{RESULTS}

Statistics were computed for the solution differences of each day. The mean, standard deviation and root mean square of the differences were derived to quantify any overall bias and variation from the benchmark solutions. In addition, the largest absolute difference in position from the benchmark solution was also noted. The average over all days and the worst case values are presented in Table 1. 
Table 1. Average and worst case values of maximum difference, mean, standard deviation and root-mean-square statistics for all solutions. Number in parentheses indicates solution day; omission of number indicates statistic is representative of several days.

\begin{tabular}{|c|c|c|c|c|c|c|c|c|c|c|c|c|c|}
\hline & \multicolumn{4}{|c|}{$\begin{array}{c}\text { Latitude } \\
\text { Difference (m) }\end{array}$} & \multicolumn{4}{|c|}{$\begin{array}{c}\text { Longitude } \\
\text { Difference (m) }\end{array}$} & \multicolumn{4}{|c|}{$\begin{array}{c}\text { Height } \\
\text { Difference (m) }\end{array}$} \\
\hline & & $\max$ & mean & $\mathrm{sd}$ & rms & $\max$ & mean & $\mathrm{sd}$ & $\mathrm{rms}$ & $\max$ & mean & $\mathrm{sd}$ & $\mathrm{rms}$ \\
\hline \multirow[t]{2}{*}{ Alts } & average & 0.33 & 0.01 & 0.07 & 0.07 & 0.18 & -0.01 & 0.04 & 0.04 & 0.99 & 0.10 & 0.23 & 0.26 \\
\hline & wor & $0.62^{(7)}$ & 0.04 & 0.11 & 0.11 & $0.28^{(7)}$ & 0.02 & 0.05 & 0.06 & $1.32^{(10)}$ & $0.24^{(3)}$ & $0.32^{(10)}$ & $0.38^{(10)}$ \\
\hline \multirow[t]{2}{*}{ WAAS } & average & 0.35 & 0.00 & 0.06 & 0.06 & 0.23 & 0.01 & 0.04 & 0.04 & 0.78 & -0.26 & 0.22 & 0.35 \\
\hline & worst & $0.58^{(7)}$ & 0.03 & 0.09 & 0.09 & $0.35^{(7)}$ & 0.03 & 0.05 & 0.05 & $0.98^{(10)}$ & $0.47^{(8)}$ & $0.29^{(10)}$ & $0.52^{(8)}$ \\
\hline \multirow[t]{2}{*}{ NATO } & average & 0.20 & 0.01 & 0.05 & 0.05 & 0.11 & -0.01 & 0.03 & 0.03 & 0.77 & 0.24 & 0.14 & 0.28 \\
\hline & worst & $0.37^{(7)}$ & 0.03 & 0.07 & 0.08 & $0.15^{(7)}$ & 0.01 & 0.04 & 0.04 & $1.09^{(10)}$ & $0.35^{(10)}$ & $0.22^{(10)}$ & $0.42^{(10)}$ \\
\hline \multirow[t]{2}{*}{ UNB1 } & average & 0.10 & 0.00 & 0.02 & 0.03 & 0.06 & 0.00 & 0.02 & 0.02 & 0.45 & 0.08 & 0.10 & 0.15 \\
\hline & worst & $0.15^{(7)}$ & 0.02 & 0.04 & 0.04 & $0.10^{(10)}$ & 0.01 & 0.02 & 0.03 & $0.71^{1}$ & $0.20^{(1}$ & $0.16^{(10)}$ & $0.26^{(1}$ \\
\hline
\end{tabular}

As we might expect, we found low biases in the latitude and longitude components of position for all the models. However the UNB1 solutions show a consistently smaller variation over the other three. The variations, as represented by the standard deviation and r.m.s. were larger for the Altshuler and proposed WAAS model. The general trends are clearly represented by Table 1 . The largest latitude and longitude variations almost all occur on 7th March. This is due to data gaps at very low elevation angles, where the use of the C/A code pseudoranges still results in large PDOP spikes.

The largest effects are seen in the height differences. The NATO and WAAS solutions have an average mean of similar magnitude, but opposite sign. Altshuler and UNB1 have similar average means. However, the variations from the benchmark solution, as represented by the standard deviation and r.m.s., are much smaller for UNB1 indicating that on average, this model causes the smallest variations from the benchmark solution.

To get a better understanding of the performance of each model, we need to study an individual solution. Because it represents the worst case for the height component we will use the data from 10th March. Figure 1 shows the variations with latitude, longitude and height for each model's solution with reference to the benchmark solution. The latitude and longitude plots use the same scale. The height difference is represented on a larger scale (approximately 5 times larger) to indicate the greater variation seen in the height component.

These figures show the generally poor performance of the Altshuler and proposed WAAS models with height as compared to the UNB1 and NATO models. The most important point to be made is that there is a "step" visible in the WAAS solution differences at 1500 metres. This appears in all the solutions computed with this model and is due to a discontinuity in the formulation of the algorithm.

In general the NATO and UNB1 models perform more consistently at all altitudes, although a diverging trend is visible at very low altitudes (below approximately $1 \mathrm{~km}$ ). The Altshuler model performance is generally poor at all times. In the worst case, it is possible to have an error of $1.32 \mathrm{~m}$ in height by using the Altshuler model. The worst case for the proposed WAAS and NATO models is approximately one metre.

The NATO model has a slight bias at all altitudes. It performs slightly worse at very low altitudes. Removing the bias would not necessarily improve its performance compared to the UNB1 model, because its variation is slightly greater. Close examination of the algorithms reveals slight discontinuities between the functions comprising the model, however they do not appear to show up in the solution differences.

The UNB1 model solution more closely approaches the benchmark solution with increasing height. The NATO model performs similarly. This indicates that the Standard Atmosphere more closely approximates the actual atmosphere with increasing height, which is what we would expect. Any tropospheric delay model should therefore perform better with increasing altitude; the fact that Altshuler and WAAS do not appear to do so is a cause for concern. 

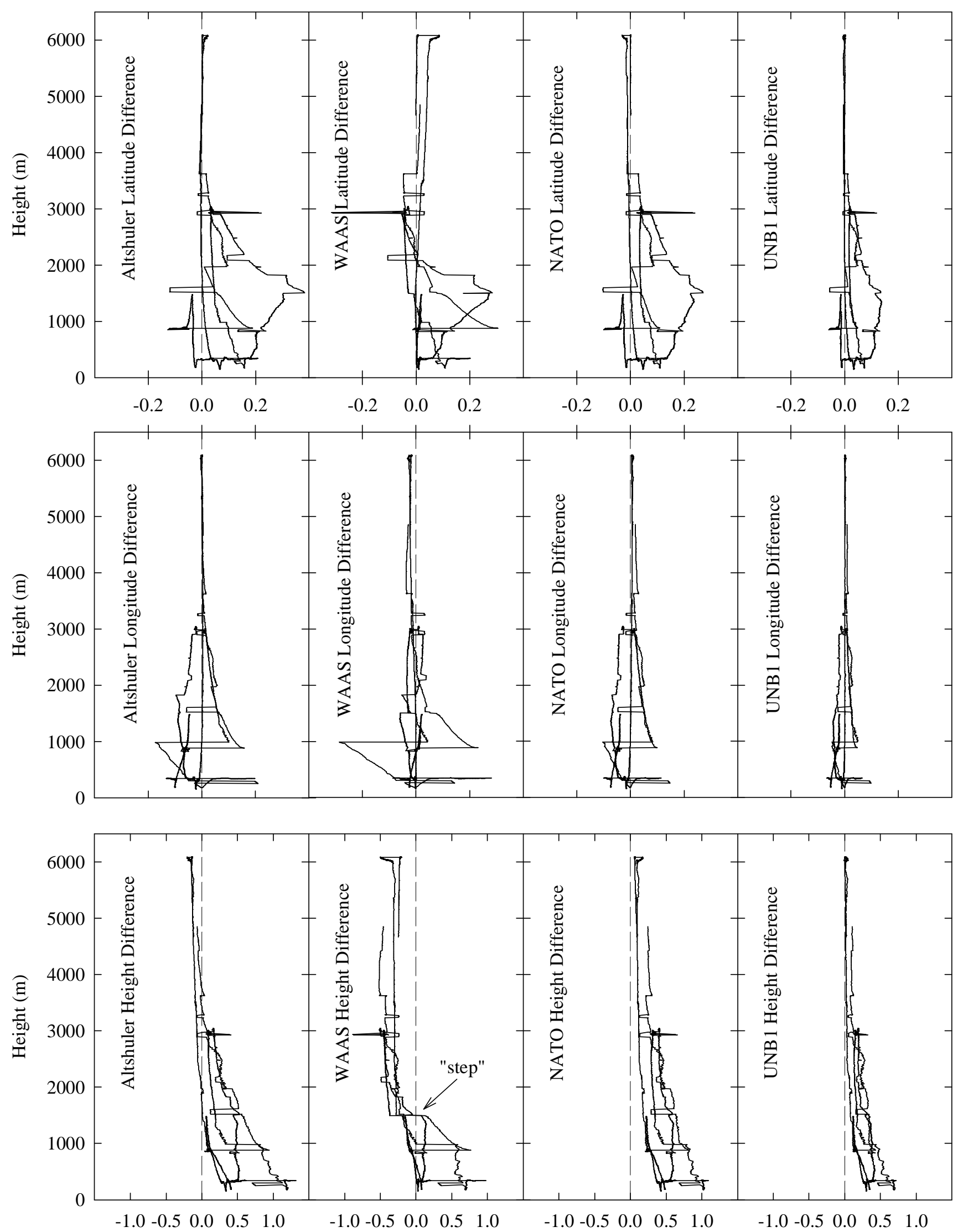

Figure 1. Solution differences for 10th March plotted against height of aircraft. All x-axis units are metres. 


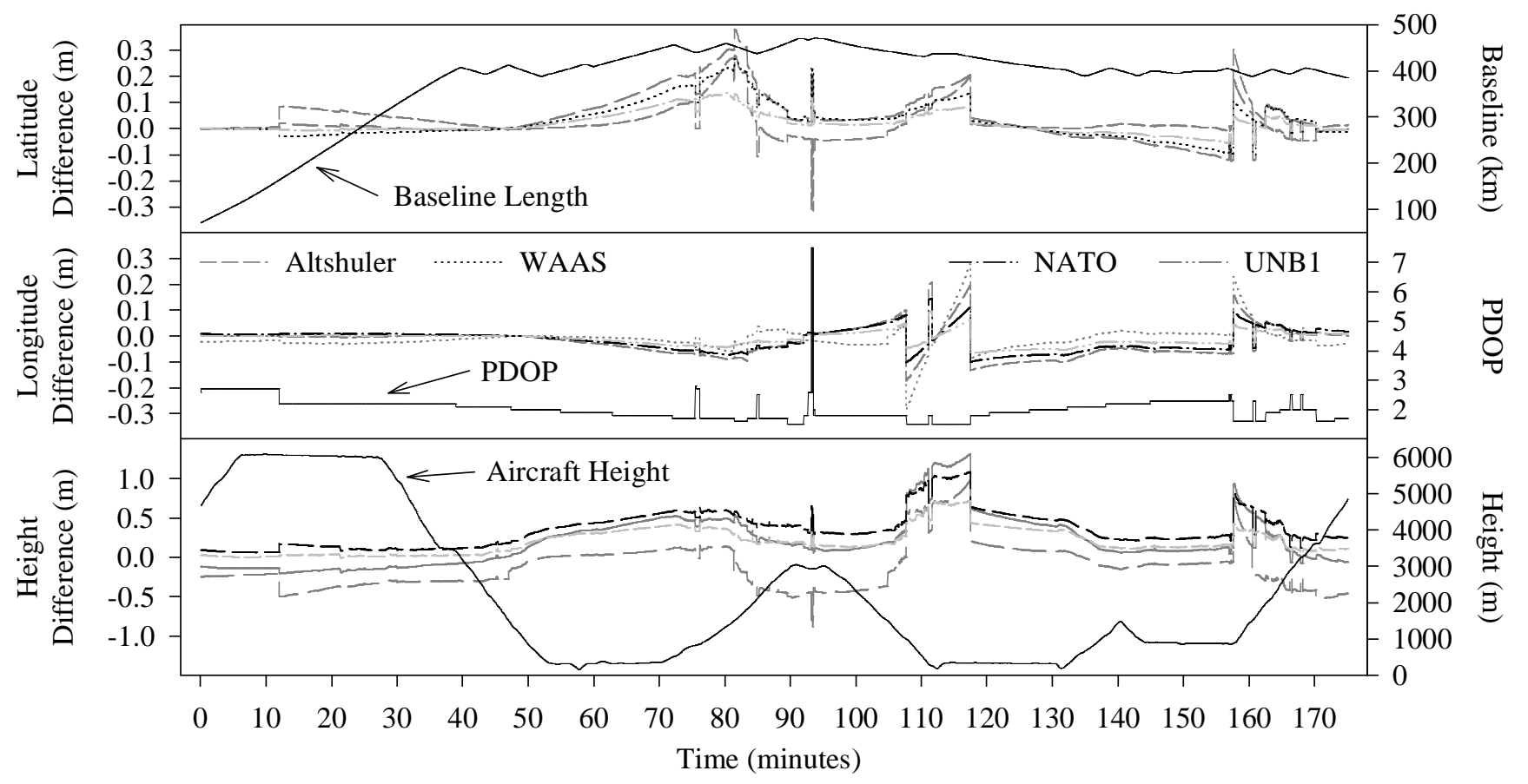

Figure 2. Solution differences for 10th March.

All of the solutions suffer to some extent from large steps and jumps. By considering Figure 2, where the solution differences are plotted against time, we can see that these are correlated with changes in the solution PDOP value. This indicates the influence of rising and setting satellites on the modelling of the tropospheric delay. These plots show that at low elevation angles, the position errors increase until the satellite sets below the elevation angle cut-off (e.g. at approximately 118 minutes in Figure 2) whereupon the solution improves. When a new satellite rises (e.g. at approximately 12 minutes in Figure 2) an error is introduced into the solution and it jumps away from the benchmark solution. As the satellite continues to rise the error decreases and the solution improves. The jumps between 100 and 120 minutes are complicated by the fact that satellites are both rising and setting at the same time and also suffer from data gaps. It should be noted that the largest latitude difference in the WAAS solution is due to a PDOP spike, however there are differences of a similar magnitude that are not. Despite the problems of these jumps they are present in all the solutions and yet are much less severe in the UNB1 solutions. Therefore, given the good performance of the Niell functions, these large trends are almost certainly due to mapping function errors in the other tested models.

\section{CONCLUSIONS}

A comprehensive investigation has been undertaken into the influence of several tropospheric delay models of the types commonly used in airborne GPS navigation. A comparison has been performed with a geodetic-type model combination using Standard Atmosphere values of pressure, temperature and water vapour pressure and standard algorithms describing their variation with height.

Based on our investigation, it would appear that the currently proposed WAAS model performs somewhat poorly compared to the Altshuler model. This is to be expected, as it is an approximation to it. Solutions computed using the proposed WAAS model with the aircraft data have a step at a height of 1500 metres. This is due to an over simplification in the formulation of the algorithm. Otherwise, above 1500 metres it models the rate of change of the delay in the atmosphere quite well.

The UNB1 model performs best overall. It consistently shows the smallest bias and variation in the solutions computed using it. Of all the models examined here, it has the most elegant formulation of the algorithms. The delay algorithms are continuous with height and are not modelled in layers such as the proposed WAAS or NATO models. The Altshuler model is described by several complex polynomials and does not accurately model the change of the atmosphere with height.

An important error source in the tested models is in the mapping functions. These are significant in Altshuler and the proposed WAAS models; the NATO model performs slightly better because of its use of the Chao mapping function. Because the same mapping functions are used in both the UNB1 and the benchmark solutions, the errors seen in these differences are the errors in the zenith delays mapped to the lower elevation angles. 
The high variation in values of the meteorological parameters close to the earth's surface are likely to be the limiting factors in mitigating the tropospheric delay errors in precise airborne GPS navigation. However, the use of standard geodetic-type zenith delay models and mapping functions, coupled with suitable surface-meteorological values should, based on our investigation, perform better than the other tropospheric delay models discussed here.

\section{REFERENCES}

[1] Altshuler, E.E. and P.M. Kalaghan (1974). "Tropospheric range error corrections for the NAVSTAR system." Interim scientific report, Air Force Cambridge Research Laboratories, Bedford, Mass., 16 April, AFCRL-TR-74-0198, 13 pp.

[2] Bean, B.R. and G.D. Thayer (1959). "Models of the atmospheric radio refractive index." Proceedings of the IRE, Vol. 47, No. 5, May, pp. 740-755.

[3] Chao, C.C. (1972). "A model for tropospheric calibration from daily surface and radiosonde balloon measurements." JPL Technical Memorandum 391350, Jet Propulsion Laboratory, Pasadena, CA.

[4] DeCleene, B. (1995). Personal communication, Federal Aviation Administration, May 1995.

[5] Davis, J.L., T.A. Herring, I.I. Shapiro, A.E.E. Rogers and G. Elgered (1985). "Geodesy by radio interferometry: Effects of atmospheric modelling errors on estimates of baseline length." Radio Science, Vol. 20, No. 6, pp. 1593-1607.

[6] Essen, L. and K.D. Froome (1951). "The refractive indices and dielectric constants of air and its principal constituents at 24,000 Mc/s." Proceedings of the Royal Society B, Vol. 64, pp. 862-875.

[7] Ifadis, I.M. (1993). "Space to earth techniques: Some considerations on the zenith wet path delay parameters." Survey Review, Vol. 32, No. 249, pp. 130-144.

[8] Janes, H.W., R.B. Langley and S.P. Newby (1991). "Analysis of tropospheric delay predicition models: Comparisons with ray-tracing and implications for GPS relative positioning." Bulletin Géodésique, Vol. 65, pp. 151-161.

[9] Langley, R.B. (1996). "Propagation of the GPS Signals." in GPS for Geodesy, International School, Delft, The Netherlands, 26 March - 1 April, 1995. Springer-Verlag, New York.

[10] Loh, R. (1993). "FAA Wide Area Integrity and Differential GPS Program." Proceedings of 2nd Internation Symposium on Differential Satellite Navigation Systems (DSNS 93), 29 March - 2 April.

[11] Loh, R., V. Wullschleger, B. Elrod, M. Lage, F. Haas (1995). "The U.S. Wide-Area Augmentation System (WAAS)." Navigation, Journal of The Institute of Navigation, Vol. 42, No. 3, Fall 1995.
[12] Mendes, V.B. and R.B. Langley (1994). "A comprehensive analysis of mapping functions used in modeling tropospheric propagation delay in space geodetic data." Proceedings of KIS94, International Symposium on Kinematic Systems in Geodesy, Geomatics and Navigation, Banff, Canada, 30 August - 2 September, pp. 87-98.

[13] Mendes, V.B. and R.B. Langley (1995). "Zenith wet tropospheric delay determination using prediction models: Accuracy analysis." Cartografia e Cadastro, Instituto Português de Cartografia e Cadastro, No. 2, pp. 41-47.

[14] Mendes, V.B., J.P. Collins and R.B. Langley (1995). "The effect of tropospheric propagation delay errors in airborne GPS precise positioning." Proceedings of ION GPS-95, Palm Springs, CA, 12 - 15 September 1995, pp. 1681-1689.

[15] NATO (1993). Standardization Agreement (STANAG) Doc. 4294 EL (Edition 1), Appendix 6 to Annex A. pp. A-6-34 - A-6-37. North Atlantic Treaty Organization, Brussels.

[16] NOAA (1976). "U.S. Standard Atmosphere 1976". NOAA S/T 76 - 1562, NTIS AD/A035 728, National Technical Information Service, U.S. Dept. of Commerce, Springfield, VA.

[17] Niell, A.E. (1996). "Global mapping functions for the atmosphere delay at radio wavelengths." Journal of Geophysical Research, Vol. 101, No. B2, pp 32273246.

[18] Owens, J.C. (1967). "Optical refractive index of air: Dependence on pressure, temperature and composition." Applied Optics, Vol. 6, No. 1, pp. 5159.

[19] Saastamoinen, J. (1972). “Atmospheric correction for the troposphere and stratosphere in radio ranging of satellites." in The Use of Artificial Satellites for Geodesy, Geophys. Monogr. Ser., Vol. 15, AGU, Washington, D.C.

[20] Shi, J. and M.E. Cannon (1995). "Critical error effects and analysis in carrier phase-based airborne GPS positioning over large areas." Bulletin Géodésique, Vol. 69, No. 4, pp. 261-273.

[21] Smith, W.L. (1966). "Note on the relationship between total precipitable water and surface dew point." J. Appl. Meteor., Vol. 5, October, pp. 726-727.

[22] Thayer, G.D. (1974). "An improved equation for the radio refractive index of air." Radio Science, Vol. 9, No. 10, pp. 803-807.

[23] Tiemeyer, B., M.E. Cannon, G. Lachapelle and G. Schänzer (1994). "Satellite navigation for high precision aircraft navigation with emphasis on atmospheric effects." Proceedings of PLANS'94, IEEE, Las Vegas, Nevada, 11-15 April, pp. 394-401. 